\title{
Perfil de sensibilidade a antibióticos de um isolado de Providencia rettgeri proveniente de equino
}

\author{
Antibiotic sensitivity profile in isolate of Providencia rettgeri from a horse
}

\author{
Luiz Eduardo Lacerda ${ }^{1}$, Ricardo Wagner Portela ${ }^{2 *}$ \\ ${ }^{1}$ Mestre em Biotecnologia. Doutorando do Programa de Pós graduação em Processos Interativos de Órgãos e \\ Sistemas. Técnico em Microbiologia. Instituto de Ciências da Saúde. Universidade Federal da Bahia - UFBA; ${ }^{2}$ Doutor \\ em Bioquímica. Professor Associado. Instituto de Ciências da Saúde. Universidade Federal da Bahia - UFBA
}

\begin{abstract}
Resumo
Introdução: a meningite bacteriana em equinos é uma enfermidade frequente em animais jovens. Streptococcus spp., Staphylococcus aureus e Escherichia coli são as bactérias mais comumente isoladas nesses casos. Apesar da bactéria Providencia rettgeri já ter sido isolada em casos de meningite humana e de crocodilo, não há relatos de seu isolamento em equinos. Objetivo: relatar o isolamento e a identificação da bactéria $P$. rettgeri de um potro com sintomas neurológicos e avaliar o perfil de sensibilidade a antibióticos deste isolado. Metodologia: o isolamento foi realizado a partir do líquido cefalorraquidiano do potro, por meio de cultivo em meio ágar chocolate. Após isolamento, as colônias formadas foram identificadas por equipamento Biotyper, baseado em espectrometria de massa. O perfil de sensibilidade foi definido por teste de difusão em discos, seguindo metodologia relatada pelo CLSI M2-A8 em 2003, sendo a bactéria classificada como resistente, padrão indeterminado ou sensível aos antibióticos, de acordo com o descrito pelo EUCAST em 2021. Resultados: este é o primeiro relato do isolamento de $P$. rettgeri como agente etiológico de meningite em potro. Dos 15 antibióticos testados, a bactéria foi resistente a 9, sensível a 5 e com padrão indeterminado a 1 antibiótico. Conclusão: nossos resultados indicam que $P$. rettgeri deve ser considerada entre possíveis agentes etiológicos de quadros neurológicos em equinos e que testes de sensibilidade a antibiótico são fundamentais, uma vez que essa bactéria já apresenta resistência a diversos antibióticos disponíveis comercialmente.
\end{abstract}

Palavras-chave: Equidae. Meningite. Antibiograma. Resistência bacteriana. Antimicrobiano.

\begin{abstract}
Introduction: Bacterial meningitis in horses is a frequent disease in young animals. Streptococcus spp., Staphylococcus aureus and Escherichia coli are the most commonly isolated bacteria in these cases. Although Providencia rettgeri bacterium has already been isolated in cases of human and crocodile meningitis, there are no reports of its isolation in cases of meningitis in horses. Objective: to report isolation and identification of the P. rettgeri bacteria from a foal with neurological symptoms and to assess antibiotic sensitivity profile in isolate of it. Methods: isolation was performed from the foal's cerebrospinal fluid, through cultivation in chocolate agar medium. After isolation, formed colonies were identified by Biotyper equipment, based on mass spectrometry. Sensitivity profile was verified by disk diffusion test, according to methodology that was reported by CLSI M2-A8 in 2003, which classified bacteria as resistant, indeterminate pattern or sensitive to antibiotics, as described by EUCAST in 2021. Results: this is the first report on isolation of $P$. rettgeri as an etiologic agent of meningitis in foals. Among 15 antibiotics that were tested, results showed bacteria resistence to 9 antibiotics, bacteria sensitivity to 5, but undetermined pattern to 1 antibiotic. Conclusion: results indicate that $P$. rettgeri shall be considered among potential etiologic agents of neurological conditions in horses and that antibiotic sensitivity tests are essential, since this type of bacterium is already resistant to several commercially available antibiotics.

Keywords: Equidae. Meningitis. Antibiogram. Bacterial drug resistance. Anti-infective agents.
\end{abstract}

\section{INTRODUÇÃO}

A meningite de origem bacteriana em equinos é mais frequente em animais jovens do que em adultos (LAUGIER; TAPREST; FOUCHER, 2009). Usualmente, essa doença deriva de complicações oriundas de uma septicemia neonatal e está associada a altas taxas de mortalidade (KOTERBA; BREWER; TARPLEE, 1984).

Vários microrganismos já foram encontrados cau-

Correspondete/Corresponding: *Ricardo Wagner Portela - End: Av. Reitor Miguel Calmon, s/n, Canela, Salvador-BA, 40231-300-Tel: (71) 98882-4660 -E-mail: rwportela@gmail.com sando meningite em equinos, sendo Streptococcus spp., Staphylococcus aureus e Escherichia coli as bactérias mais comumente isoladas nessas infeç̧ões (FURR, 2015). No entanto, existe uma diferença entre agentes infecciosos documentados nas infecções em potros e em cavalos. Nos adultos, predominam as espécies estreptocócicas, já em potros predominam as bactérias Gram-negativas, pois estas estão associadas aos agentes causadores de septicemia neonatal, que resultam em meningites nesses animais, sendo a $E$. coli a bactéria mais comum nessas infecções de equinos jovens (VIU et al., 2012).

Não há relatos do isolamento da bactéria Providencia rettgeri a partir de meningite em equinos; no entanto, 
este bacilo Gram-negativo já foi isolado em casos de meningite em humanos, apesar de ser incomum (MAITI; SINGH; PANDEY, 2013), e de meningite em crocodilos recém-nascidos de água salgada (Crocodylus porosus) (LADDS; BRADLEY; HIRST, 1996). Em humanos, já foi relatada como responsável por infecções do trato urinário e por septicemia (CHO et al., 2010; KIM et al., 2003). Em equinos, essa bactéria já foi encontrada em infecções de feridas (COCHRANE et al., 2009) e como agente primário não convencional de diarreia em potros (OLIVo et al., 2016).

As espécies de Providencia podem ser amplamente resistentes a antibióticos, apresentando genes de resistência a diversos antibióticos (OLAITAN et al., 2016). Stock e Wiedemann (1998) relataram a susceptibilidade natural aos antibióticos em cepas de $P$. rettgeri, descrevendo resistência a tetraciclinas e fosfomicina e apresentando mais susceptibilidade a quinolonas, aminoglicosídeos, fosfomicina e antibióticos beta-lactâmicos, quando comparada a Providencia stuartii (STOCK; WIEDEMANN, 1998). Maiti, Singh e Pandey (2013) avaliaram a susceptibilidade a antibióticos de $P$. rettgeri presentes em três casos de infecções incomuns do sistema nervoso central em humanos, percebendo que as cepas isoladas apresentaram resistência a ampicilina, ceftriaxona, cefotaxima e ceftazidima (cefalosporinas de terceira geração), ciprofloxacino-ofloxacino e piperacilina, no entanto, demonstraram sensibilidade apenas ao imipenem (MAITI; SINGH; PANDEY, 2013).

Devido à extensa resistência natural a antibióticos e ao isolamento recente de cepas multirresistentes de $P$. rettgeri, atenção especial deve ser dada à escolha da terapêutica de antibióticos para tratamento adequado das infecções provocadas por elas. Assim, o objetivo deste trabalho foi relatar o isolamento e a identificação da bactéria $P$. rettgeri de um potro com sintomas neurológicos e avaliar o perfil de sensibilidade a antibióticos deste isolado.

\section{METODOLOGIA}

\section{Avaliação clínica do animal}

A avaliação clínica do potro foi realizada por uma análise do histórico do animal, anamnese e exame clínico geral e específico do sistema nervoso. 0 exame específico do sistema nervoso foi conduzido para se detectar as alterações neurológicas, sendo avaliado o comportamento, estado mental, postura, andadura, propriocepção, inspeção geral para evidenciar possível assimetria, além da avaliação da função dos nervos cranianos, conforme descrito por Furr e Reed (2015).

\section{Coleta do material clínico}

Como se trata de uma afecção neurológica, o material clínico coletado foi o líquido cefalorraquidiano (LCR). A coleta foi realizada através da centese da cis- terna cérebro-medular (espaço atlanto-occipital), com o animal sedado, em posição quadrupedal. A amostra foi conservada sob refrigeração por 48 horas, até a análise microbiológica.

\section{Isolamento do microrganismo}

$\mathrm{O}$ isolamento do microrganismo foi realizado a partir do LCR coletado do potro. Com uma alça calibrada estéril de $10 \mathrm{ul}$, foi retirada uma alíquota do líquor e realizado o esgotamento por estria em placa contendo ágar chocolate. A placa foi incubada a $37^{\circ} \mathrm{C}$ por 24 horas, em condições aeróbicas.

\section{Identificação do microrganismo por MALDI TOF}

As colônias obtidas no ágar chocolate tiveram identificação realizada em equipamento Biotyper (Bruker Daltonics, Billerica, MA), baseado em MALDI TOF MS. Após cultivo em ágar BHI (infusão de cérebro e coração) por 24 horas a $37^{\circ} \mathrm{C}$, uma colônia isolada foi aplicada em um poço da placa de aço alvo MALDI Biotyper. Adicionou-se ao poço $1 \mu \mathrm{l}$ de ácido fórmico (70\%) e $1 \mu \mathrm{l}$ de matriz MALDI-TOF MS (composta por uma solução saturada de ácido $\alpha$-ciano-4-hidroxicinâmico), deixando-se secar em temperatura ambiente (ASSIS et al., 2017; RIFICl et al., 2020).

Para análise, usou-se um espectrômetro de massa FlexControl MicroFlex LT (Bruker Daltonics), equipado com um laser de nitrogênio de $20 \mathrm{~Hz}$. Os espectros foram analisados usando o software Bruker Biotyper 3.0 e a biblioteca versão 3.3.1.0 (4.613 entradas). Antes que as medições da amostra fossem registradas, o espectrômetro foi calibrado usando-se um padrão de teste bacteriano (E. coli DH5 alfa). Pontuações de corte recomendadas pelo fabricante foram usadas para identificação, com pontuações $\geq 2.000$, indicando identificação em nível de espécie, enquanto pontuações $\geq 1,700$ e $<2.000$ indicam identificação em nível de gênero e pontuações $<1.700$ não indicam uma identificação confiável (ASSIS et al., 2017; RIFICl et al., 2020).

\section{Perfil de sensibilidade a antimicrobianos}

O perfil de sensibilidade foi definido através de teste de difusão em discos, seguindo metodologia adaptada do CLSI M2-A8 (2003) realizada em duplicata. Utilizaram-se discos disponíveis comercialmente, cada um impregnado com um antibiótico, sendo então testados 15 antimicrobianos: amicacina (AMI 30 - marca: Sensifar), ampicilina (AMP 10 - Bio-Rad), aztreonam (ATM 30 Laborclin), bacitracina (BAC 10 - Sensifar), cefalotina (CFL 30 - Sensifar), ceftriaxona (CRO 30 - Laborclin), ciprofloxacino (CIP 05 - Sensifar), cloranfenicol (CLO 30 Sensifar), doxiciclina (DOX 30 - Sensidisc), enrofloxacina (ENO 05 - Sensidisc), gentamicina (GEN 10 - Sensifar), neomicina (NEO 30 -Sensifar), nitrofurantoina (NIT 300 - Sensifar), norfloxacino (NOR 10 - Sensifar) e sulfazo- 
trim (SUT 25 - Laborclin). Esses foram escolhidos por serem antibióticos de amplo espectro ou de espectro reduzido para bactérias Gram-negativas e que podem ser utilizados para tratamento de meningite.

Para o teste de difusão em discos, a bactéria foi inicialmente cultivada em ágar BHI (infusão de cérebro e coração) a $37^{\circ} \mathrm{C}$ por 24 horas. Após esse período, foi realizada a preparação do inóculo, retirando-se colônias da placa e adicionando-as em $5 \mathrm{~mL}$ de soro fisiológico estéril, até a solução atingir uma turbidez óptica comparável à da solução padrão 0,5 da escala McFarland.

$O$ inóculo foi então semeado na superfície de ágar Mueller-Hinton (Acumedia) com um swab estéril, cobrindo toda a superfície da placa. Após cinco minutos, tempo para a absorção do inóculo, foram adicionados, com o auxílio de uma pinça estéril, os discos impregnados com os antibióticos. As placas foram invertidas 15 minutos após a colocação dos discos e incubadas a $37^{\circ} \mathrm{C}$ por 24 horas, em estufa microbiológica.

Após a incubação, mediram-se os diâmetros dos halos de inibição e o tamanho foi comparado aos padrões descritos no EUCAST (2021), sendo então a bactéria classificada em resistente ao antibiótico (quando há uma grande probabilidade de falha terapêutica, mesmo quando há aumento da exposição), indeterminado (quando há uma alta probabilidade de sucesso terapêutico pelo aumento da exposição ao agente, seja pelo ajuste do regime de dosagem ou da concentração no local da infecção) ou sensível (quando há uma alta probabilidade de sucesso terapêutico, usando um regime de dosagem padrão do agente).

Os antibióticos cefalotina, bacitracina, doxiciclina e enrofloxacina não apresentam pontos de corte padrão estabelecidos pela EUCAST (2021); assim, para cefalotina e bacitracina, a não formação de halo de inibição caracterizou a bactéria como resistente. Em relação à doxiciclina e à enrofloxacina, o tamanho do halo foi comparado com padrão estabelecido pelo CLSI (2020).

\section{RESULTADOS}

A partir do líquido cefalorraquidiano do potro apresentando meningite e sintomas neurológicos, isolou-se apenas uma cepa bacteriana. Ela apresentava bom crescimento em ágar chocolate, após 24 horas de cultivo em $37^{\circ} \mathrm{C}$, em condições aeróbicas, apresentando-se como colônias médias, branco-acinzentadas, com aspecto mucoide e brilhoso, com bordas irregulares (Figura 1). Após coloração de Gram, observaram-se bastonetes Gram-negativos isolados e/ou aos pares. Por Biotyper, a bactéria isolada foi identificada como Providencia rettgeri. A identificação realizada caracterizou-se por um alto grau de certeza de identificação de espécie e pela ausência de contaminantes.
Figura 1-Providencia rettgeri em ágar chocolate após 24 horas de cultivo à $37^{\circ} \mathrm{C}$ em condições aeróbicas.

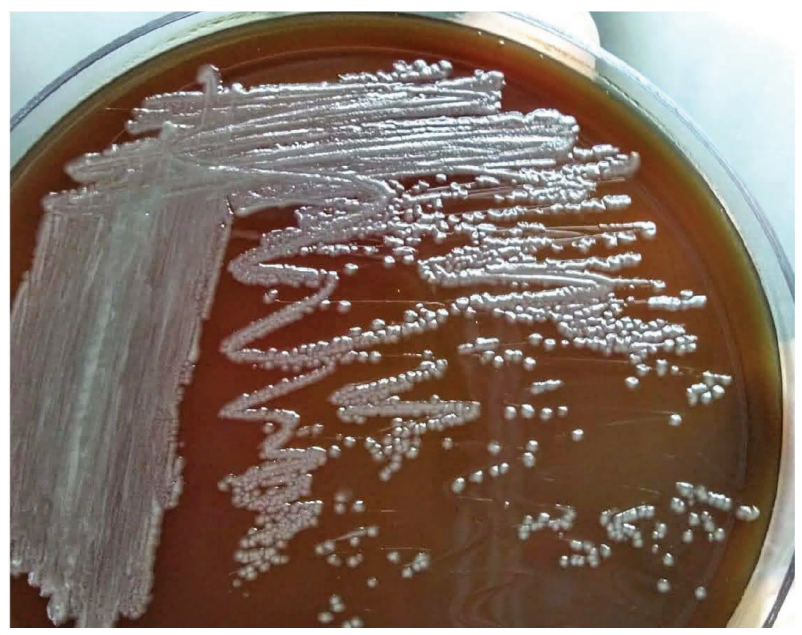

Fonte: Dados da pesquisa.

Após o teste de difusão em disco, realizado em duplicata e seguindo as recomendações da CLSI M2-A8 (2003), e com análise dos resultados seguindo o descrito pela EUCAST (2021) e pelo CLSI (2020), para a doxiciclina e enrofloxacina, identificou-se, de forma qualitativa, que dentre os 15 antibióticos testados, a cepa estudada é resistente a 9 antibióticos, sensível a 5 e tem padrão indeterminado a 1 antibiótico, o aztreonam (Tabela 1).

Tabela 1-Resultado do teste de difusão em discos, em duplicata (placas A e B), para avaliação da sensibilidade de Providencia rettgeri a antibióticos.

\begin{tabular}{|c|c|c|c|}
\hline Antibiótico & $\begin{array}{c}\text { Diâmetro } \\
\text { de halo } \\
\text { (mm) }\end{array}$ & Resultado & $\begin{array}{l}\text { Ponto de corte do } \\
\text { diâmetro de halo } \\
\text { (mm) pela } \\
\text { EUCAST, } 2021\end{array}$ \\
\hline Amicacina & (A) 28, (B) 30 & Sensível & $(\mathrm{S})>18(\mathrm{R}) \leq 18^{\mathrm{b}}$ \\
\hline Ampicilina & Sem halo & Resistente & $(\mathrm{S})>14(\mathrm{R})<14^{\mathrm{b}}$ \\
\hline Aztreonam & (A) 23, , (B) 23 & Indeterminado & $(\mathrm{S}) \geq 26$ (I) $25-21(\mathrm{R})<21$ \\
\hline Bacitracina & Sem halo & Resistente & Sem dados ${ }^{a}$ \\
\hline Cefalotina & Sem halo & Resistente & Sem dados ${ }^{a}$ \\
\hline Ceftriaxona & Sem halo & Resistente & $(\mathrm{S}) \geq 25$ (I) $24-22(\mathrm{R})<22$ \\
\hline Ciprofloxacina & (A) 35, (B) 33 & Sensível & $(S) \geq 25$ (I) $24-22$ (R) $<22$ \\
\hline Cloranfenicol & Sem halo & Resistente & $(S)>17(R) \leq 17^{b}$ \\
\hline Doxiciclina & (A) $10,(B) 10$ & Resistente & $(S) \geq 14(R)<14^{b c}$ \\
\hline Enrofloxacina & (A) 22, (B) 22 & Resistente & $(S) \geq 23(R)<23^{b c}$ \\
\hline Gentamicina & (A) 25, (B) 22 & Sensível & $(\mathrm{S}) \geq 17(\mathrm{R})<17^{\mathrm{c}}$ \\
\hline Neomicina & (A) 14, (B) 13 & Sensível & $(\mathrm{S})>12(\mathrm{R}) \leq 12^{\mathrm{c}}$ \\
\hline Nitrofurantoina & Sem halo & Resistente & $(\mathrm{S}) \geq 11(\mathrm{R})<11^{\mathrm{c}}$ \\
\hline Norfloxacina & (A) 30, (B) 31 & Sensível & $(\mathrm{S})>22(\mathrm{R}) \leq 22^{\mathrm{c}}$ \\
\hline Sulfazotrim & Sem halo & Resistente & $(\mathrm{S})>14$ (I) $14-11(\mathrm{R})<11$ \\
\hline
\end{tabular}

Fonte: Dados da pesquisa.

Legenda: S-Sensivel. $R$-Resistente. ${ }^{a}$-Sem ponto de corte estabelecido pela EUCAST (2021). ${ }^{b}$ - Ponto de corte estabelecido pelo CLSI (2020). ${ }^{c}$ Sem ponto de corte estabelecido pela EUCAST (2021) para classificação como Indeterminado.

Nota: O teste foi conduzido de acordo com o recomendado pelo CLSI (2003), e a avaliação dos dados foi realizada seguindo orientações da EUCAST (2021) e do CLSI (2020). 
Dos 5 antibióticos para os quais o isolado de $P$. rettgeri apresentou sensibilidade, 3 são da classe dos aminoglicosídeos (amicacina, gentamicina e neomicina), 1 é da classe das quinolonas (ciprofloxacino) e 1 é uma fluoroquinolona (norfloxacino), sendo todos de largo espectro. A bactéria apresentou resistência à ampicilina (beta-lactâmico), à bacitracina (polipeptídio), cefalotina e ceftriaxona (cefalosporinas de primeira e de terceira geração, respectivamente), cloranfenicol (anfenicol), doxiciclina (tetraciclina), nitrofurantoina (nitrofurano), sulfazotrim (sulfanilamida) e à enrofloxacina (fluoroquinolona).

\section{DISCUSSÃO}

A bactéria Providencia rettgeri é considerada como patogênica, com relato de infecções em humanos (principalmente do trato urinário e casos de septicemia) e animais. A meningite por esse microrganismo é incomum (LADDS; BRADLEY; HIRST, 1996; MAITI; SINGH; PANDEY, 2013), sendo descrito seu isolamento apenas em humanos e em crocodilos recém-nascidos. O presente estudo é o primeiro relato desse tipo de infecção em equinos, o que indica que a bactéria deve ser considerada entre possíveis agentes etiológicos de meningite nesses animais.

Por $P$. rettgeri ser uma bactéria Gram-negativa, é muito possível que a infecção neurológica tenha começado com uma sepse, uma vez que, como está descrito na literatura, casos de meningite em potros causada por bactérias Gram-negativas estão usualmente associados à septicemia neonatal (VIU et al., 2012). No entanto, como não foi realizada análise microbiológica do sangue, não se tem evidências que confirmem essa hipótese.

A cepa de $P$. rettgeri estudada apresentou sensibilidade aos aminoglicosídeos (amicacina, gentamicina e neomicina), a quinolonas (ciprofloxacina) e a uma das fluoroquinolonas (norfloxacina) testadas. Esse resultado concorda com os de Stock e Wiedemann (1998), que perceberam boa susceptibilidade natural da espécie a essas classes de antibióticos.

No entanto, quando se fala em terapêutica contra a meningite, não se deve observar apenas a sensibilidade do microrganismo ao antibiótico, mas também se este atinge concentrações antibacterianas no líquido cefalorraquidiano, pois nem todas as drogas antimicrobianas conseguem ultrapassar a barreira hematoencefálica. A inflamação meníngea pode aumentar a proporção de antibiótico que pode cruzar essa barreira, mas isso é variável e pode não ser adequado para resultar em eficácia terapêutica. Em muitos casos, pode ser indicada a terapia combinada de antibióticos ou a injeção direta de antimicrobiano no líquido cefalorraquidiano (administração intratecal), de forma combinada ou não (LACOMBE; FURR, 2015).

Apesar de a cepa estudada ter sido sensível aos aminoglicosídeos, eles não são absorvidos por via oral, devido à estrutura policatiônica altamente polar, e por administração parenteral não atingem concentrações significativas no líquido cefalorraquidiano de equinos, devido a sua fraca permeabilidade à barreira hematoencefálica (DOWLING, 2004; SOUSA, 2006). Apenas se consegue atingir concentrações eficazes desses antibióticos no líquor, recorrendo-se a uma administração intratecal (com exceção da neomicina) (SOUSA, 2006). Além disso, outro problema relacionado a essa classe de antibióticos é a sua nefrotoxicidade potencial, fato já relatado em potros (RIVIERE et al., 1983). Esses aspectos dificultam seu uso no tratamento da meningite, sendo pouco recomendados para esse caso. Quinolonas e fluoquinolonas, por sua vez, atravessaram a barreira hematoencefálica, alcançando altas concentrações no sistema nervosos central, após administração parenteral (COTTAGNOUD; TÄUBER, 2003; HASBUN; QUAGLIARELLO, 2000), sendo excelente para o tratamento contra a cepa isolada no presente estudo.

Segundo Stock e Wiedemann (1998), um isolado de $P$. rettgeri estudado por eles apresentou sensibilidade natural ao aztreonam (beta-lactâmico). Para a cepa isolada no potro, esse antibiótico apresentou um padrão indeterminado de sensibilidade, ou seja, existe alta probabilidade de sucesso terapêutico, com o aumento da exposição a ele, seja pelo ajuste do regime de dosagem ou da concentração no local da infeç̧ão. Assim, é provável que com esse ajuste seja possível utilizá-lo para o tratamento de meningite causada pela cepa isolada do potro, sendo uma alternativa ao uso dos aminoglicosídeos (LENTNEK; WILLIAMS, 1991; PAXSON; PARADIS, 2010).

Todos os antibióticos para os quais o isolado de $P$. rettgeri apresentou sensibilidade são de largo espectro. $\mathrm{O}$ uso de antibióticos de espectro amplo pode ser arriscado, pois pode favorecer a resistência a antimicrobianos, se utilizados inadvertidamente e de maneira incorreta (DORON; DAVIDSOM, 2011), podendo ser necessária a busca de um novo antimicrobiano para o tratamento dessa bactéria daqui a alguns anos.

Dos antibióticos testados, a ceftriaxona, cloranfenicol, enrofloxacina e sulfazotrim são altamente recomendados para o tratamento de meningite bacteriana em equino (FURR, 2015) e essa cepa bacteriana foi resistente a todas. Sem o isolamento dessa bactéria e teste de sensibilidade, o tratamento usual não seria efetivo contra esse microrganismo.

\section{CONCLUSÃO}

O encontro de $P$. rettgeri como causador de meningite em equinos é importante, indicando que essa bactéria deve ser considerada pela comunidade veterinária entre possíveis agentes etiológicos de quadros neurológicos desses animais. Nossos resultados indicam que aminoglicosídeos e quinolonas podem ser boas escolhas para tratar infecções por $P$. rettgeri, mas, no caso de uma meningite, o tratamento mais simples seria com quinolonas, pois os aminoglicosídeos não atingem boas concentrações no líquido cefalorraquidiano por via parenteral, sendo necessária a administração intratecal. 
É recomendado que a escolha do antibacteriano para tratamento adequado de meningite seja determinado por cultura e teste de sensibilidade de uma amostra do líquido cefalorraquidiano. Isto porque muitos microrganismos podem ser responsáveis por meningite nos equinos, mas também porque, mesmo sabendo dos efeitos bactericidas dos aminoglicosídeos e quinolonas sobre a $P$. rettgeri, esta bactéria apresenta extensa resistência natural a diversos antibióticos e, recentemente, tem sido descrito o isolamento de cepas multirresistentes, existindo a possibilidade da cepa causadora da infecção ser resistente aos antibióticos dessas classes.

\section{AGRADECIMENTOS}

Agradecemos a Carolina Ferreira Amorim, mestranda do Programa de Pós - graduação em Microbiologia, da Universidade Federal da Bahia, pela ajuda na preparação e execução do antibiograma e compilação dos resultados; a Tatiana Figueiredo Paiva, médica veterinária, pelo manuseio do animal e coleta do material biológico; e a Roselane Gonçalves Santos, doutoranda em Bioinformática, da Universidade Federal de Minas Gerais, pela ajuda na identificação do microrganismo por MALDI TOF.

\section{REFERÊNCIAS}

ASSIS, G. B. N. et al. Use of MALDI-TOF mass spectrometry for the fast identification of Gram-positive fish pathogens. Front. Microbiol., [s.I.], v. 8, p. 1492, 2017.

$\mathrm{CHO}, \mathrm{H}$. et al. A case of Providencia rettgeri sepsis in a patient with cervical cord injury. Infect. Chemother., Tokyo. v. 42, n. 6, p. 428-430, 2010.

CLSI. Padronização dos testes de sensibilidade a antimicrobianos por disco-difusão: Norma Aprovada - Oitava Edição (M2-A8). Pensilvânia: NCCLS, 2003. 58 p.

CLSI. Performance standards for antimicrobial disk and dilution susceptibility tests for bacteria isolated from animals (VETO1S). $5^{\text {th }} \mathrm{ed}$. Pennsylvania: NCCLS, 2020. 56 p.

COCHRANE, C. A. et al. Biofilm evidence and the microbial diversity of horse wounds. Can. J. Microbiol., Ottawa, v. 55, n. 2, p. 197-202, 2009.

COTTAGNOUD, P.; TÄUBER, M. G. Fluoroquinolones in the treatment of meningitis. Curr. Infect. Dis. Rep., [s.I.], v. 5, p. 329-336, 2003.

DORON, S.; DAVIDSON, L. E. Antimicrobial stewardship. Mayo Clin. Proc., Rochester, v. 86, n. 11, p. 1113-1123, 2011.

DOWLING, P. M. Antimicrobial drugs. In: BERTONE, J. J.; HORSPOOL, L. J. I. (Eds.). Equine clinical pharmacology. Philadelphia: Saunders, 2004. p. 13-48.

EUCAST. Breakpoint tables for interpretation of MICs and zone diameters. European Committee on Antimicrobial Susceptibility Testing. Versão 11.0. 2021. Disponível em: https://www.eucast.org/fileadmin/ src/media/PDFs/EUCAST_files/Breakpoint_tables/v_11.0_Breakpoint_ Tables.xlsx. Acesso em: 01 ago. 2021.
FURR, M. Bacterial infections of the central nervous system. In: FURR, M.; REED, S. (Eds.). Equine neurology. $2^{\text {nd }}$ ed. Oxford: Blackwell, 2015. p. 273-282.

FURR, M.; REED, S. Examination of the Nervous System. In: FURR, M.; REED, S. (Ed.). Equine neurology. $2^{\text {nd }}$ ed. Oxford: Blackwell. 2015, p. 67-78.

HASBUN, R.; QUAGLIARELLO, V. J. Use of the quinolones in treatment of bacterial meningitis. In: ANDRIOLE, V. T. (Ed.). The quinolones. $3^{\text {rd }}$ ed. Massachusetts: Academic Press, 2000. p. 325-342.

KIM, B. et al. Bacteremia due to tribe protease: A review of 132 Cases during a decade (1991-2000). Scand. J. Infect. Dis., Stockholm, v. 35, p. 98-103, 2003.

KOterba, A. M.; BReWeR, B. D.; TARPLEe, F. A. Clinical and clinicopathological characteristics of the septicemic neonatal foal: review of 38 cases. Equine Vet. J., London, v. 16, p. 376-382, 1984.

LACOMBE, V. A.; FURR, M. Pharmaceutical considerations for treatment of central nervous system disease. In: FURR, M.; REED, S. (Ed.). Equine neurology. $2^{\text {nd }}$ ed. Oxford: Blackwell, 2015. p. 46-48.

LADDS, P. W.; BRADLEY, J.; HIRST, R. G. Providencia rettgeri meningitis in hatchling saltwater crocodiles (Crocodylus porosus). Aurt. Vet., [s.I.], v. 74, n. 5, p. 398-398, 1996.

LAUGIER, C. et al. A necropsy survey of neurologic disease in 4,319 horses examined in Normandy (France) from 1986 to 2006. J. Equine Vet. Sci., [s.l.], v. 29, p. 561-568, 2009.

LENTNEK, A. L.; WILLIAMS, R. R. Aztreonam in the treatment of Gramnegative bacterial meningitis. Rev. Infect. Dis., Chicago, v. 13, supl. 7, p. S586-S590, 1991.

MAITI, T. K.; SINGH, V. K.; PANDEY, P. Providencia rettgeri: an unusual cause of central nervous system infections. Am. J. Med. Sci.,Hagerstown, v. 346, n. 2, p. 158-159, 2013.

OLAITAN, A. O. et al. Genomic plasticity of multidrug-resistant NDM-1 positive clinical isolate of Providencia rettgeri. Genome Biol. Evol., [s.I.], v. 8, n. 3, p. 723-728, 2016.

OLIVO, G. et al. Enteric pathogens and coinfections in foals with and without diarrhea. BioMed Res. Int., [s.I.], v. 2016, 12 p., 2016.

PAXSON, J. A.; PARADIS, M. R. Pharmacokinetics of aztreonam after intravenous administration in foals. J. vet. Pharmacol. Therap., [s.I], v. 34, p. 92-94, 2010.

RIFICI, C. et al. Atypical multibacterial granulomatous myositis in a horse: first report in Italy. Vet. Sci., [s.I], v. 7, n. 2, p. 47, 2020.

RIVIERE, J. E. et al. Species dependent gentamicin pharmacokinetics and nephrotoxicity in the young horse. Fundam. Appl. Toxicol., Orlando, v. 3, p. 448-457, 1983.

SOUSA, J. C. Manual de antibióticos antibacterianos. 2. ed. Porto: Edições Universidade Fernando Pessoa, 2006. 686 p.

STOCK, I.; WIEDEMANN, B. Natural antibiotic susceptibility of Providencia stuartii, $P$. rettgeri, P. alcalifaciens and $P$. rustigianii strains. J. Med. Microbiol., [s.l.], v. 47, p. 629-642, 1998.

VIU, J. et al. Clinical findings in 10 foals with bacterial meningoencephalitis. Equine Vet. J., London, v. 44, p. 100-104, 2012.

Submetido em: 19/11/2021

Aceito em: 20/11/2021 